\title{
Of Rags and Ragulator
}

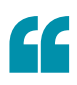

Ragulator ... is responsible for mTORC 1 translocation

The mammalian target of rapamycin complex 1 (mTORC1) kinase regulates cell growth in response to growth factors, energy levels and amino acid availability, and is activated by RHEB, a Ras-related GTP-binding protein. Rag GTPases are amino acid-specific regulators of the mTORC1 pathway, but how they regulate mTORC1 was unknown. However, it was proposed that amino acids promote the translocation of mTORC1 to the surface of an endomembrane compartment in a Rag-dependent manner, where mTORC1 can colocalize with its activator, RHEB. A new study shows that mTORC1 moves to lysosomal membrane surfaces in response to amino acid stimulation, and that a protein complex called Ragulator, which interacts with Rag GTPases, is responsible for mTORC1 translocation and is necessary for its amino acid-dependent activation.

Imaging studies revealed that the presence of amino acids drives mTORC1 translocation to lysosomal membranes, where the Rag GTPases reside. This amino acid-induced movement is Rag-dependent, as it was eliminated by RNA interferencemediated Rag knockdown. By contrast, mTORC1 translocation does not depend on growth factor stimulation, RHEB or mTORC1 activity.
But how are Rag GTPases targeted to lysosomes? Protein purification approaches led to the identification of three proteins - MAPK scaffold protein 1 (MP1), p14 and p18 that form a complex and have orthologues in Drosophila melanogaster. The complex, Ragulator, co-immunoprecipitates with Rag GTPases, and p18 interacts directly with Rag GTPases in vitro. In cells lacking p14 or p18, endogenous Rag GTPases no longer localize to lysosomes, but their lysosomal localization can be reconstituted by the presence of wildtype Ragulator subunits. Also in these cells, the lysosomal recruitment of mTORC1 in response to amino acid stimulation is defective. In p18-null cells, expression of a modified p18 protein that is targeted to mitochondria leads to the mitochondrial localization of Rag GTPases. Together, these findings suggest that $\mathrm{p} 18$ is sufficient to target Rag GTPases to their subcellular location, and that the Ragulator-Rag complex serves as an amino acid-regulated docking site for mTORC1 on lysosomal membranes.

Loss of p14 and p18 function in mammalian and D. melanogaster cells prevented amino acid stimulation from activating TORC1 signalling. Interestingly, when mTORC1 was constitutively targeted to lysosomal

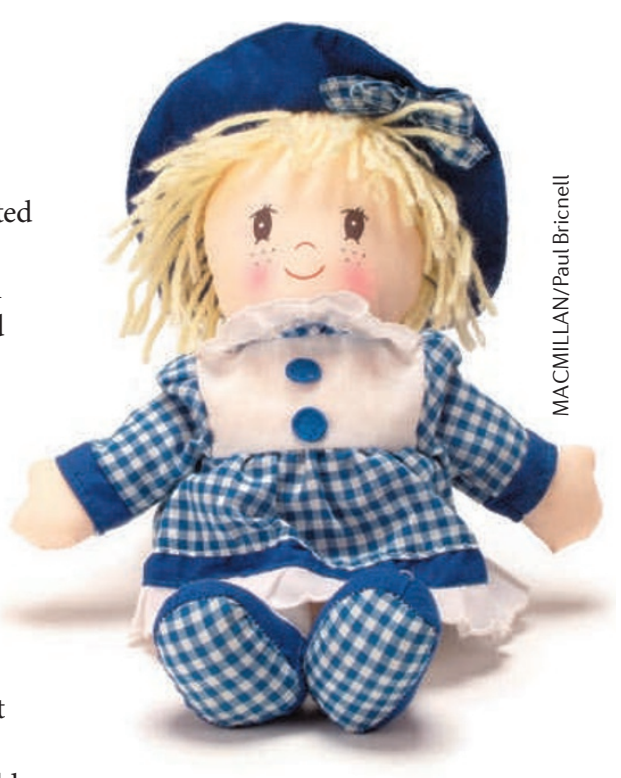

membranes, the pathway became insensitive to amino acid stimulation or deprivation and independent of Rag and Ragulator, but not RHEB, function.

These data identify Ragulator as a new, crucial component of the TORC1 signalling pathway in mammals and flies. The location of the Rag GTPases, Ragulator and mTORC1 on the lysosomal surface implicates this organelle as being important for amino acid sensing in an as yet unknown manner.

Arianne Heinrichs, Chief Editor,

Nature Reviews Molecular Cell Biology

ORIGINAL RESEARCH PAPER Sancak, Y.et al. Ragulator-Rag complex targets mTORC1 to the lysosomal surface and is necessary for its activation by amino acids. Cell 141, 290-303 (2010) 